\title{
Incidence of second primary cancers in North Portugal—a population-based study
}

\author{
Luís Pacheco-Figueiredo ${ }^{1,2,3}$ • Luís Antunes ${ }^{4}$. \\ Maria José Bento ${ }^{4}$ Nuno Lunet ${ }^{1,2}$ (D)
}

Received: 24 August 2014 / Accepted: 22 May 2015 /Published online: 10 July 2015

(C) Springer Science+Business Media New York 2015

\begin{abstract}
Purpose Longitudinal studies are needed to characterise the burden of second primary malignancies among cancer survivors. Therefore, we quantified the incidence rate and cumulative incidence of second primary cancers (SPC) and standardised incidence ratios (SIR) in a population-based cohort of subjects diagnosed with a first primary cancer (FPC). Methods We evaluated a cohort of cancer patients from the Portuguese North Region Cancer Registry (RORENO), with the first diagnosis in 2000-2003 ( $n=39451)$, to estimate the incidence rate and cumulative incidence of SPC and standardised incidence ratios (SIR), for different periods of follow-up, up to 5 years; SPC were defined according to the International Association of Cancer Registries and the International Agency for Research on Cancer guidelines.

Results The incidence rate of SPC was more than 5-fold higher in the first 2 months of follow-up than in the period between 2 months and 5 years (metachronous SPC), across which the incidence rates were relatively stable. Cancer survivors had an overall higher incidence rate of cancer than the
\end{abstract}

Nuno Lunet

nlunet@med.up.pt

1 Department of Clinical Epidemiology, Predictive Medicine and Public Health, University of Porto Medical School, Porto, Portugal

2 EPIUnit-Institute of Public Health, University of Porto (ISPUP), Porto, Portugal

3 Life and Health Sciences Research Institute (ICVS), School of Health Sciences, University of Minho, Braga, Portugal

4 North Region Cancer Registry (RORENO), Portuguese Oncology Institute, Porto, Portugal general population $(\mathrm{SIR}=1.31(95 \%$ confidence interval $(\mathrm{CI})$, 1.25-1.38)), although that difference faded when only metachronous SPC were considered (SIR=1.02 (95\% CI, 0.96-1.08)). Cancer incidence rates were higher among female lung FPC survivors and lower in prostate FPC cancer survivors than in the general population. The 5-year cumulative risk of developing a metachronous SPC was 3.0\% and reached nearly $5.0 \%$ among patients with $\mathrm{FPC}$ associated with lower risk of death.

Conclusions Cancer survivors had higher incident rates of cancer that the general population, especially due to diagnoses in the first months following the FPC. Nevertheless, after this period SPC remain frequent events among cancer survivors. Implications for cancer survivors SPC constitute an important dimension of the burden of cancer survivorship, and this needs to be taken into account when defining strategies for surveillance, prevention and counselling.

Keywords Neoplasms $\cdot$ Second primary $\cdot$ Population-based cancer registry $\cdot$ Incidence $\cdot$ Mortality

\section{Introduction}

The increasing number of incident cases of cancer [1] and the improvements in survival [2] have been contributing to a growing population of subjects with a previous diagnosis of cancer. It was estimated that in 2012, there were more than 32 million cancer survivors worldwide [3]; these subjects have an increased risk of several adverse health events and use of health resources [4], including the recurrence of the first primary cancer (FPC), cardiovascular diseases or second primary cancers (SPC) [5]. 
The occurrence of SPC is mostly related to genetic characteristics, persistence of deleterious environmental exposures and late effects of FPC treatment [6]. Recognising frequent FPC-SPC pairs can also be a useful starting point for investigating possible shared etiologies and mechanisms of carcinogenesis. Quantifying and characterising the risk of second malignancies can have important implications for surveillance, prevention and counselling.

SPC represented $16.0 \%$ of all incident cancer cases in USA (2003) [5], $6.3 \%$ in Europe (1995-1999) [7] and $3.8 \%$ in North Portugal (2000-2003) [8]. These differences in the frequency of SPC reflect the heterogeneous distribution of cancer incidence and survival across settings, as well as the distinct sensitivity of populationbased cancer registries with different operating times for detecting SPC in cross-sectional analyses [7]. A longitudinal assessment is expected to contribute to more accurate estimates, and a better understanding of the burden of SPC, by providing more clinically relevant measures of incidence.

Therefore, we followed for 5 years in order a populationbased cohort of cancer survivors to estimate incidence rates of SPC and corresponding standardised incidence ratios (SIR), which reflect the dynamics of SPC diagnosis and their relation with the expected cancer incidence in the general population. Cumulative incidences of SPC were computed to quantify the absolute risk of cancer survivors being diagnosed with another primary cancer.

\section{Methods}

\section{Study population and design}

We conducted a cohort analysis based on the North Region Cancer Registry (RORENO). This population-based cancer registry (RORENO) was set up in 1988 and covers the whole northern region of Portugal, corresponding to approximately 3.3 million inhabitants, which is nearly one third of the Portuguese population.

All cases of cancer, other than skin non-melanoma, registered in the period 2000-2003, were followed for 5 years, until the diagnosis of a new primary cancer or until death, whichever occurred first; the vital status of the cancer patients was assessed through the National Health System database.

Tumour topography and morphology were classified according to the International Classification of Diseases for Oncology, Third Edition (ICD-O-3) categories, and then recoded to the International Statistical Classification of Diseases and Related Health Problems 10th Revision (ICD-10).

\section{Definition of multiple primary cancers}

To define multiple primary cancers (MPC), we followed the guidelines proposed by the International Association of Cancer Registries (IACR) and the International Agency for Research on Cancer (IARC) [9]. Briefly, these criteria consider primary cancers those that originally developed in an organ or tissue, not being an extension, a recurrence or a metastasis. Different morphologies (even with a same topography) or dissimilar topographies should be regarded as MPC, regardless of the time between the diagnoses, unless they correspond to systemic cancers, which are considered the same cancer.

Whenever more than two primary cancers were observed in the same patient, only the second primary cancer (SPC) was considered; third and subsequent primary cancers were disregarded for the present analysis.

We classified the SPC as synchronous when diagnosed within 2 months of the corresponding FPC [10].

\section{Statistical analysis}

Person-years at risk (PYAR) among people diagnosed with a FPC was calculated as the time from the diagnosis of the FPC until 5 years of follow-up, date of death or date of diagnosis of a SPC, whichever came first.

The incidence rate of SPC was computed for different periods (" 0 to $<2 \mathrm{~m}$ "- - from the diagnosis until less than 2 months of follow-up; " $\geq 2$ to $<12$ m"-from 2 months until less than 12 months of follow-up; " $\geq 12$ to $\leq 60 \mathrm{~m}$ "-from 12 to 60 months of follow-up) since the diagnosis of the FPC, by dividing the incident cases of SPC by the PYAR within each time interval. Incidence rates were estimated also for women and men and for the most frequent types of FPC.

Standardised incidence ratios (SIRs) and corresponding $95 \%$ confidence intervals $(95 \% \mathrm{CI})$, derived from the Poisson distribution, were computed to evaluate age-adjusted ratios of cancer incidence rates between cancer survivors and the general population. The SIRs were calculated dividing the observed number of SPC by the expected number of cancer cases in the same period of time, if the cancer incidence rates in the general population had been observed among cancer survivors. The latter were estimated multiplying the PYAR among cancer survivors by the sex- and age group-specific cancer incidence rates observed in the general population of North Portugal in 2006 [11].

We computed the cumulative incidence of metachronous SPC and all-cause death for up to 5 years, stratified by sex and the most frequent types of FPC. Cumulative incidence was calculated by dividing the observed number of cases of SPC or deaths until each period of analysis $(2,6,12,24,36,48$ and 
60 months of follow-up) by the total number of survivors in the beginning of the study.

All analyses were conducted using STATA $^{\circledR}$, version 9.2 (StataCorp LP, College Station, Texas, USA).

\section{Results}

Among the 39,451 patients with a FPC in 2000-2003, a total of 1589 SPC were observed in the first 5 years after the diagnosis of the FPC (120473 PYAR), from which 417 $(26.2 \%)$ were diagnosed within the first 2 months after the FPC (synchronous tumours), 281 (17.7\%) between 2 or more months and less than 1 year and 891 (56.1\%) between 1 and 5 years.

A total of $61.0 \%$ of the SPC were observed among men and $56.6 \%$ in subjects aged 65 years or more. Regarding the distribution according to the most common groups of SPC, in relation with the observed for FPC diagnosed in 2000-2003, during the 5-year follow-up, there was a lower proportion of SPC of the female breast (6.8 vs. $12.4 \%$ ), prostate (8.1 vs. $11.9 \%$ ) and stomach (9.2 vs. $11.1 \%$ ) and a higher proportion of cancer of the colon and rectum (13.3 vs. $10.0 \%$ and 7.9 vs. $5.7 \%$, respectively), lung (10.7 vs. $7.9 \%$ ) and bladder (6.2 vs. $5.0 \%$ ) (Table 1$)$.

\section{Incidence rate of SPC and SIR}

Overall, the incidence rate of SPC was more than 5-fold higher in the first 2 months of follow-up than in the period between 2 months and 5 years, across which the incidence rates were relatively stable. However, the differences between the 0 - to $<2-\mathrm{m}$ and the $\geq 2$ - to $\leq 60$-m periods were greater among the individuals with stomach, colon, rectum and lung FPC than among those with breast, prostate or bladder FPC (Fig. 1).

The incidence rates observed among cancer survivors was more than $30 \%$ higher than the expected (SIR=1.31 $(95 \%$ CI, 1.25-1.38)), especially among women (SIR women $=1.73$ (95\% CI, 1.60-1.88) vs. SIR men=1.13 (95\% CI, 1.06 1.21)). This higher number of observed cases was mainly identified during the first 2 months of follow-up (synchronous SPC), and when this period was excluded from the analyses, the SIR were much lower (SIR=1.02 (95\% CI, 0.96-1.08); $\mathrm{SIR}$ women $=1.28(95 \% \mathrm{CI}, 1.17-1.41)$; SIR men $=0.91$ (95\% CI, 0.85-0.98)).

During the first 2 months of follow-up, the SIR were higher, both among women $(\mathrm{SIR}=10.22$ (95\% CI, 8.811.8)) and men $(\mathrm{SIR}=4.86(95 \% \mathrm{CI}, 4.3-5.5))$; the results were similar for the most frequent topographies of FPC (Figs. 2 and 3).
Table 1 Characteristics of the study cohort

\begin{tabular}{|c|c|c|c|c|}
\hline \multirow[t]{2}{*}{ Total } & \multicolumn{2}{|c|}{ First primary cancer (FPC) } & \multicolumn{2}{|c|}{ Second primary cancer (SPC) } \\
\hline & $N$ & $\%$ & $N$ & $\%$ \\
\hline \multicolumn{5}{|l|}{ Sex } \\
\hline Males & 21,694 & 55.0 & 970 & 61.0 \\
\hline Females & 17,757 & 45.0 & 619 & 39.0 \\
\hline \multicolumn{5}{|l|}{ Age at first diagnosis } \\
\hline $0-14$ & 307 & 0.8 & 4 & 0.3 \\
\hline $15-49$ years & 7356 & 18.6 & 213 & 13.4 \\
\hline $50-64$ years & 11,539 & 29.2 & 472 & 29.7 \\
\hline 65 years and over & 20,249 & 51.3 & 900 & 56.6 \\
\hline \multicolumn{5}{|l|}{ Follow-up interval } \\
\hline 0 to $<2$ months & n.a. & & 417 & 26.2 \\
\hline$\geq 2$ to $<12$ months & n.a. & & 281 & 17.7 \\
\hline$\geq 12$ to $<60$ months & n.a. & & 891 & 56.1 \\
\hline \multicolumn{5}{|l|}{ Topography } \\
\hline Stomach $(\mathrm{C} 16)$ & 4382 & 11.1 & 146 & 9.2 \\
\hline Colon (C18) & 3955 & 10.0 & 211 & 13.3 \\
\hline Rectum (C19 and C20) & 2240 & 5.7 & 125 & 7.9 \\
\hline Lung (C34) & 3136 & 7.9 & 170 & 10.7 \\
\hline Bladder (C67) & 1977 & 5.0 & 99 & 6.2 \\
\hline Female breast (C50) & 4894 & 12.4 & 108 & 6.8 \\
\hline Prostate (C61) & 4696 & 11.9 & 128 & 8.1 \\
\hline Other & 14,171 & 35.9 & 602 & 37.9 \\
\hline
\end{tabular}



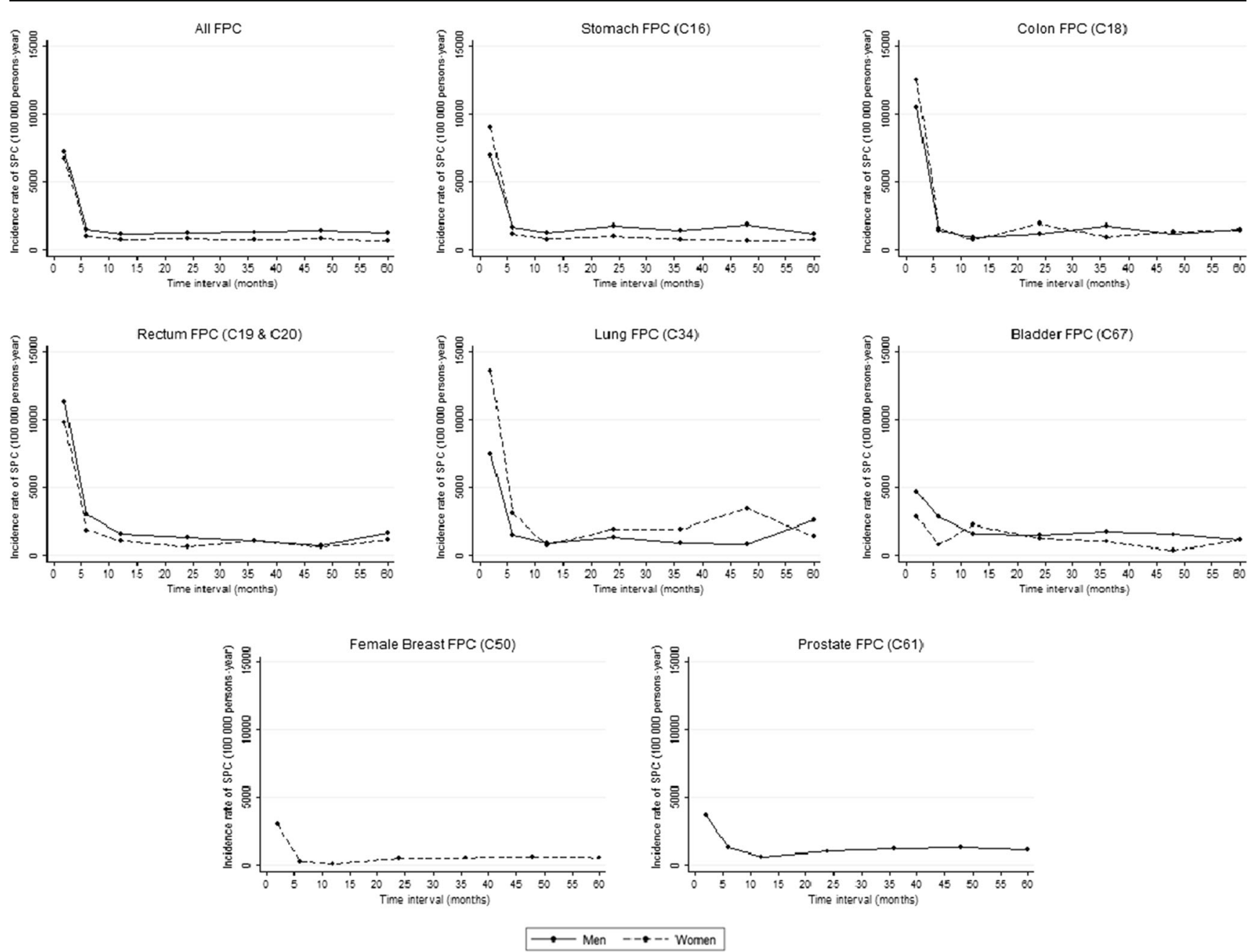

Fig. 1 Trends in incidence rates of second primary cancers (SPC) since the diagnosis of the corresponding first primary cancers $(F P C)$. Incidence rates were estimated and represented for the following intervals: " 0 to

$<2$ months"; " $\geq 2$ to $<6$ months"; " $\geq 6$ to $<12$ months"; " $\geq 12$ to $<24$ months"; " $\geq 24$ to $<36$ months"; " $\geq 36$ to $<48$ months"; " $\geq 48$ to $<60$ months"

The incidence rate among female survivors was higher than in the general population across the $\geq 2$ - to $<12$-m (SIR $=1.37$ $(95 \% \mathrm{CI}, 1.13-1.67))$ and the $\geq 12$ - to $\leq 60-(\mathrm{SIR}=1.26(95 \%$ CI, 1.13-1.40)) follow-up intervals (Fig. 2). For men, the incidence rates were similar to those observed in the general population in the same periods $(\geq 2$ to $<12$ : $\mathrm{SIR}=0.91(95 \%$ CI, $0.78-1.06) ; \geq 12$ to $\leq 60 \mathrm{~m}: \mathrm{SIR}=0.91(95 \% \mathrm{CI}, 0.84$ 0.99)) (Fig. 3).

Among women, the SIR was lower in the $\geq 2$ - to $<12$-m period for those with a breast FPC (SIR $=0.36(95 \% \mathrm{CI}$, $0.17-0.76)$ ). Those with a lung FPC were more likely to develop a SPC during the $\geq 12$ - to $\leq 60$-m period after the diagnosis of the FPC (SIR=3.00 (95\% CI, 1.47-6.11)). Among men, prostate cancer survivors showed a lower incidence rate of cancer than the general population in the $\geq 2$ to $<12-\mathrm{m}(\mathrm{SIR}=0.52(95 \% \mathrm{CI}, 0.37-0.74))$ and $\geq 12$ - to $\leq 60-\mathrm{m}(\mathrm{SIR}=0.67(95 \% \mathrm{CI}, 0.58-0.78))$ follow-up intervals. After the first 2 months of follow-up, there were no statistically significant differences in the cancer incidence rates between the general population and the cancer survivors with other FPC (Figs. 2 and 3).

\section{Cumulative incidence of SPC and death}

The 5-year cumulative incidence of SPC was $3.0 \%$ (women, $2.5 \%$; men, $3.4 \%$ ) and the risk of death in the same period was $46.1 \%$ (women, $38.8 \%$; men, $52.1 \%$ ) (Fig. 4; Appendix 1).

Women with FPC of the colon or bladder had the highest 5year risk of developing a SPC, 4.0 and $3.6 \%$, respectively, along with a 5-year cumulative risk of death among the lowest (colon, 46.2 \%; bladder, $37.8 \%$ ). Those with lung and stomach FPC had some of the lowest 5 -year risk of developing a SPC, 2.5 and $1.7 \%$, respectively, and the highest 5-year risk of death (lung, $83.2 \%$; stomach, $62.0 \%$ ). Although the female breast cancer survivors had the lowest 5 -year risk of death (19.4\%), the 5-year risk of developing a SPC was also among the lowest (2.2\%) (Fig. 5; Appendix 1). 
Fig. 2 Standardised incidence ratios $(S I R)$ and $95 \%$ confidence intervals $(95 \% \mathrm{CI})$ for the diagnosis of a second primary cancer, according to the first primary cancer and follow-up time since its diagnosis, among women

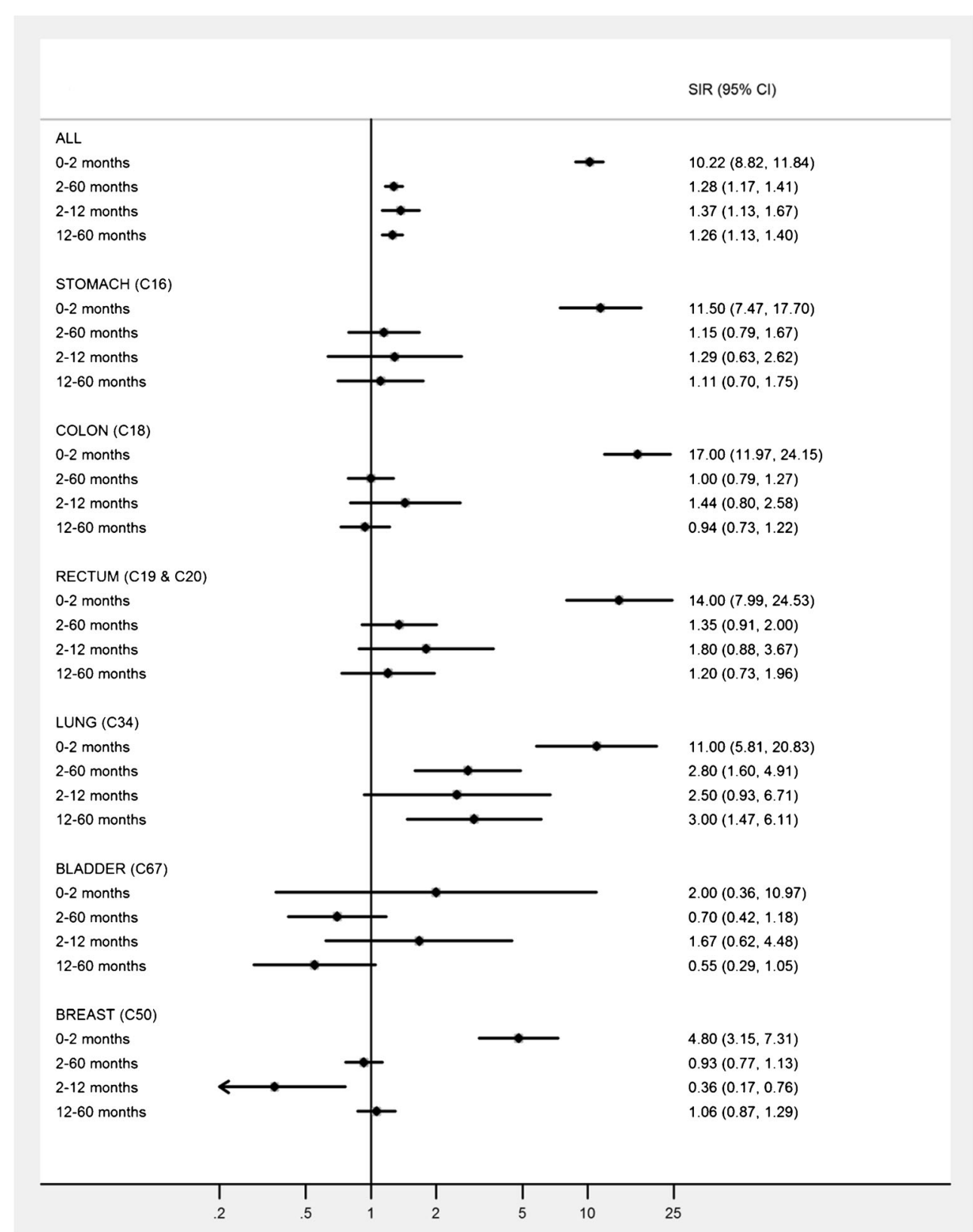

Men with prostate and bladder FPC had the highest 5-year cumulative incidence of SPC, 5.5 and $4.5 \%$, respectively, and were among those with the lowest 5-year risk of death (prostate, $25.0 \%$; bladder, $35.2 \%$ ). Subjects with a lung or a stomach FPC had the lowest 5-year risk of a SPC, 1.2 and $2.7 \%$, respectively, and were among those with highest 5 -year risk of death (lung, $87.8 \%$; stomach, $67.3 \%$ ) (Fig. 5).

\section{Discussion}

Cancer survivors had an overall higher incidence rate of cancer than the general population of North Portugal, although that difference fades when only metachronous SPC are considered. The 5-year cumulative risk of developing a SPC was $3.0 \%$ and reached nearly $5.0 \%$ among patients with FPC associated with lower risk of death.

The incidence rates of cancer were approximately $30 \%$ higher among cancer survivors, but similar to the observed in the general population when only the metachronous SPC were considered. The latter result is in agreement with a previous Finish study (SIR $=0.99$ (95 \% CI, 0.95-1.03)) [12], whereas higher incidence rates of metachronous SPC were observed in Australia (SIR=1.27 (95\% CI, 1.25-1.29)) [13], France ( $\mathrm{SIR}=1.36$ (95\% CI, 1.35-1.38)) [14], Japan $(\mathrm{SIR}=1.21(95 \% \mathrm{CI}, 1.19-1.23))[15]$ and the USA $(\mathrm{SIR}=$ 1.14 (95\% CI, 1.14-1.15)) [5], and lower rates were reported in Danish (SIR=0.91 (95\% CI, 0.89-0.93)) [16], UK (SIR = 
Fig. 3 Standardised incidence ratios (SIR) and $95 \%$ confidence intervals $(95 \% \mathrm{CI})$ for the diagnosis of a second primary cancer, according to the first primary cancer and follow-up time since its diagnosis, among men

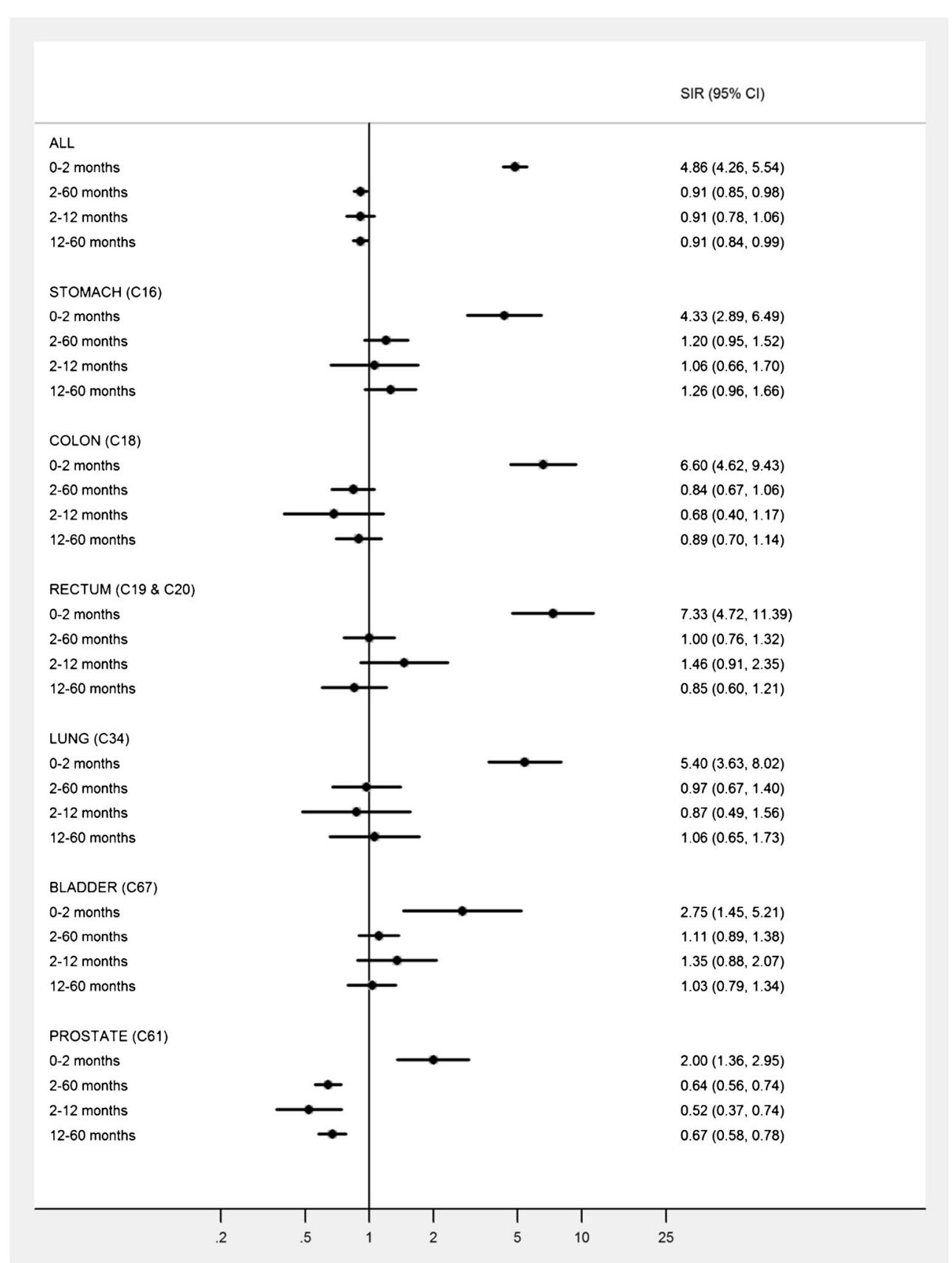

0.77 (95\% CI, 0.75-0.79)) [17] and Italian (SIR =0.93 (95\% CI, 0.90-0.96)) [18] studies. The heterogeneity of these results may reflect different patterns of cancer risk across populations and periods analysed, as well as the use of distinct definitions of SPC and synchronous tumours (intervals ranging from 2 months up to 1 year after the diagnosis of the FPC) and differences in the length of follow-up.

Including synchronous SPC in the analyses allowed the comparison of incidence rates since the diagnosis of the FPC, which is seldom presented in other population-based studies [18], and the assessment of the effect of diagnosis anticipation in the incidence rates throughout follow-up.
In our study, more than one quarter of the SPC were synchronous tumours, reflecting the strong influence of common diagnostic and staging procedures performed during the clinical management of the FPC $[19,20]$. This contributed to an increased number of SPC diagnoses that otherwise would had not been identified soon after the diagnosis of the FPC. Since the FPC topographies with the highest incidence rates of SPC in the first 2 months corresponded to the lowest incident rates in the remaining 58 months of analysis, excluding the synchronous SPC might have contributed to an underestimation of the incidence rates after the first 2 months. Among our data, we found that those FPC with more demanding staging 

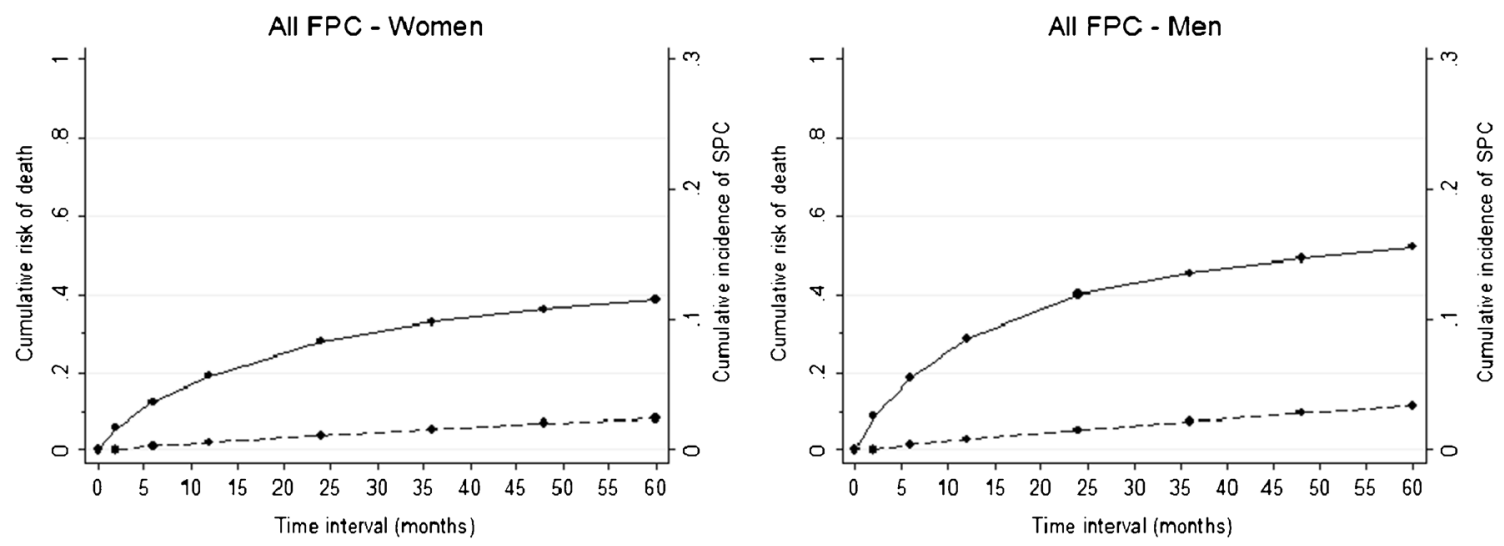

Fig. 4 Cumulative risk of death and incidence of second primary cancers $(S P C)$, among women and men. Cumulative risks of death were estimated and represented for the following intervals: " 0 to $<2$ months"; " $\geq 2$ to $<6$ months"; " $\geq 6$ to $<12$ months"; " $\geq 12$ to $<24$ months"; " $\geq 24$ to $<36$ months"; " $\geq 36$ to $<48$ months"; " $\geq 48$ to $<60$ months"; cumulative

incidences of SPC were estimated and represented for the following intervals: " $\geq 2$ to $<6$ months"; " $\geq 6$ to $<12$ months"; " $\geq 12$ to $<24$ months"; " $\geq 24$ to $<36$ months"; " $\geq 36$ to $<48$ months"; " $\geq 48$ to $<60$ months"

procedures at diagnosis (colon, rectum and lung tumours), which usually include a CT scan and an endoscopic evaluation, showed higher differences of incidence rate between the 0 - to $<2$-m and the $\geq 2$ - to $\leq 60$-m periods.

This tendency for anticipation of the diagnosis of a SPC had already been recognised in previous studies, where 20 $30 \%$ of all SPC diagnosed in the first 5 years were identified within 2 months of the incidence date of the FPC $[16,18]$. This phenomenon is analogous to the expected trend of the incidence rate after a screening test, with a strong increase at the beginning (synchronous FPC), when prevalent cases are identified through anticipation of cancer diagnosis, followed by a decrease due to the lack of diagnosis of cancers already identified; afterwards, a smooth slope of further increase until the usual incidence levels are reached again [21]. One example that illustrates the impact of excluding a high number of synchronous tumours in the quantification of metachronous SIR, was observed in the UK study [17], which reported lower SIR due to considering one of the largest synchronous periods (12 months).

On other hand, several reports had documented that cancer survivors with a more recent diagnosis had a higher SIR of SPC than those diagnosed in earlier years [13, 15]. Although the reasons for those differences are not yet completely understood, cancer screening has been increasing during the past decades [22] and is more frequent among cancer survivors than in the general population [23], which may result in higher SPC incidence rates in more recent years.

Beyond the strong influence of screening and surveillance procedures in the increased incidence rates observed during the first stages of survivorship, subtle genetic differences, FPC treatment options and previous deleterious environmental exposures should also be considered as potential determinants of the observed SIRs. Future studies with Portuguese cancer

patients, using sizable samples analysing the potential role of genetic polymorphisms, tobacco or ethanol consumption and cancer treatment options (chemotherapy agents or radiotherapy) as risk factors for the incidence of SPC among survivors to different FPC, are awaited. The change in behaviours after a cancer diagnosis should also be considered an important determinant of SPC incidence rates across different settings. A previous study from North Portugal had shown similar patterns of lifestyle exposures among cancer survivors and the general population [24], although it was not clear if this was a consequence of behavioural change or a survival bias, since the study used a cross-sectional design.

Our results showed that prostate cancer survivors had lower incidence rate of cancer than the general population, when analysing the entire follow-up period, in accordance to what was observed in other settings $[5,13,18]$. A possible explanation for those findings is the high proportion of prostate cancers diagnosed by screening, which is more likely among individuals with higher educational and socioeconomic status, and consequently with a healthier profile than the general population $[25,26]$.

Several studies have documented higher metachronous cancer incidence rates among female breast cancer survivors than in the general population $[5,13-15]$, essentially due to tighter medical surveillance [20], a strong link between hormonal-related cancers [27] and a genetic predisposition (such as BRCA1 and BRCA2 syndromes) [28, 29]. However, in our study, the rates in female breast cancer survivors were similar to that observed in the general population. A plausible explanation for this discrepancy is the high proportion (22\%) of synchronous SPC that we observed in breast cancer survivors, reflecting the anticipation of cancer diagnoses. When considering both synchronous and metachronous SPC, the 
Fig. 5 Cumulative risk of death and incidence of second primary cancers $(S P C)$, for the most frequent topographies of the first primary cancers, among women and men. Cumulative risks of death were estimated and represented for the following intervals: " 0 to $<2$ months"; " $\geq 2$ to $<6$ months"; " $\geq 6$ to

$<12$ months"; " $\geq 12$ to

$<24$ months"; " $\geq 24$ to

$<36$ months"; " $\geq 36$ to

$<48$ months"; " $\geq 48$ to

$<60$ months"; cumulative

incidences of SPC were estimated and represented for the following intervals: " $\geq 2$ to $<6$ months"; " $\geq 6$ to $<12$ months"; " $\geq 12$ to

$<24$ months"; " $\geq 24$ to

$<36$ months"; " $\geq 36$ to

$<48$ months"; " $\geq 48$ to

$<60$ months"
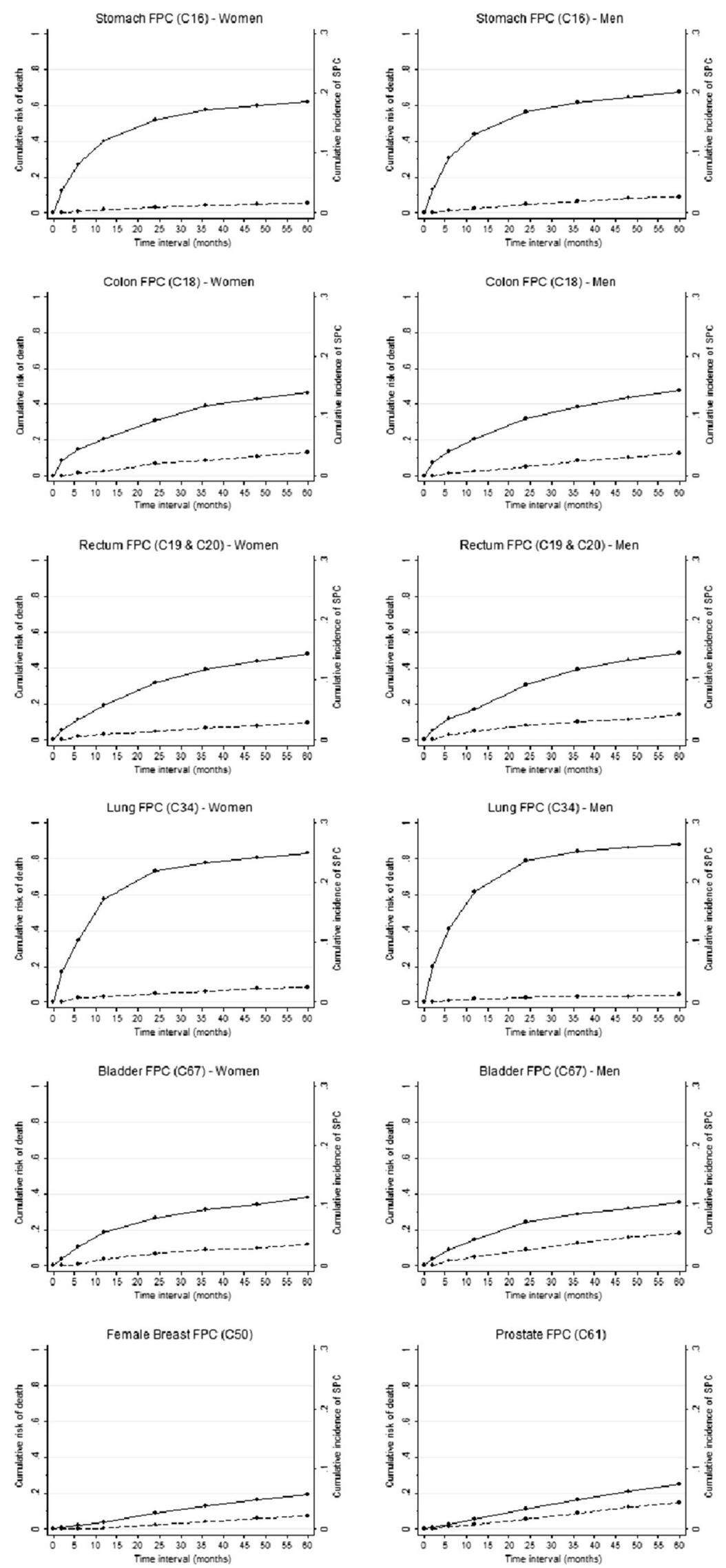

$\longrightarrow$ Death --๑-- SPC 
observed SIR was 1.09 (95\% CI, 0.91-1.29), which is closer to that observed in other studies [5]. Additionally, since a high proportion of female breast cancers are diagnosed by screening and women adhering to screening tend to be more educated than the general population [30], we may hypothesise that a large proportion of these female cancer survivors had healthier behaviour than the general population and, consequently, a lower incidence rate of a SPC. The latter could have also happened for colon and rectum FPCs, since the incidence rates of these cancers is also likely to be influenced by screening procedures.

Women with a lung FPC had a higher cancer incidence rate than the general population, when considering the entire follow-up period, although among men there were no significant differences. A lower competing risk of death among women than in men with lung cancer [31] may contribute to the differences observed. However, since in Portugal women are at an earlier stage of the tobacco epidemic than men [32, $33]$, this may reflect mostly a more pronounced contrast between women with lung cancer and those from the general population regarding the exposure to risk factors such as smoking.

The FPCs with the highest survival rates had the highest 5year cumulative incidence of SPC. As observed in other settings [5], these results are mostly likely explained by the competing risk of death that contributes to a lower probability of developing a SPC among the FPC topographies with worse survival. We restricted our follow-up evaluation to 60 months, because it was the longest time frame available with a complete dataset regarding the studied variables. As this study used data from a population-based cancer registry, this cohort of patients diagnosed with a FPC within 2000-2003, will continue to be followed up, allowing future evaluations with longer time frames.

To our knowledge, this was the first study to estimate the 5year cumulative risk of developing a subsequent cancer among cancer survivors in Portugal. The 5-year cumulative incidence estimates were lower than those from the SEER, which ranged between 2.8 and $8.9 \%$, respectively, for lung and bladder FPC [5]. However, our results show that in North Portugal, as in other settings, SPC should be regarded as a problem commonly encountered in routine medical practice, rather than a rare event. Moreover, data on the cumulative incidence of SPC provides local clinicians with a benchmark to estimate the probability of cancer survivors developing another cancer within 5 years.

Despite the strengths of using data from a population-based cancer registry, constituting an important piece of information for understanding the burden of SPC in North Portugal, some limitations need to be discussed. We only presented the overall SIR of SPC across the most frequent FPC, due to the low number of SPC in strata of less frequent FPC, precluding the identification of frequent FPC-SPC pairs, which are a useful starting point for investigating possible shared etiologies and mechanisms of carcinogenesis. Survival is already high for several cancers, and a longer follow-up is needed for a more comprehensive understanding of the burden of SPC among cancer survivors. The completeness of registration of SPC may be higher than for FPC because cancer survivors have contacted with the cancer registry sources when the FPC was identified, which could contribute to an overestimation of SIR; however, the completeness of the registry is high [34], and this is not expected to have a major impact in the SIR estimates. On the other hand, in patients already being followed, a new primary cancer may had been confused with recurrence, remaining unregistered; this is more likely to have occurred, depending on the procedures implemented in each source of data for the registry, contributing to an underestimation of the number of SPC.

The population-based nature of the data used in our analyses allows broad inference at a population level; however, the interpretation of the results is limited by the absence of data on the treatments to which each case was submitted, as well as regarding their socioeconomic, behavioural or genetic characteristics. Although high-penetrance mutations in cancer susceptibility genes, such in familial adenomatous polyposis (FAP), hereditary nonpolyposis colorectal cancer (HNPCC), BRCA1 and BRCA2 syndromes or Li-Fraumeni disease, are estimated to make only a small contribution to the SPC burden, owing to their low frequency [35], the prevailing model is that genetic risk of both first and subsequent primary cancers is defined by the cumulative effect of multiple low-penetrance and intermediate-penetrance risk alleles for cancer, where each individual genetic variant confers a modest increase in risk, but which collectively increase risk substantially when co-inherited in an individual [35]; the identification of cases likely to be due to a high genetic susceptibility would allow a better understanding of the determinants of the burden of SPC. Finally, although we did not have data regarding the stage at diagnosis of the FPC, precluding the assessment of its role along the SPC incidence rates, we expect that those survivors with lower stages at diagnosis will have higher incidence rates of SPC, due to their expectedly higher survival.

In conclusion, cancer survivors had higher incident rates of cancer than the general population, especially due to diagnoses in the first months following the FPC. Nevertheless, after this period SPC remain frequent events among cancer survivors and constitute an important dimension of the burden of cancer survivorship. This needs to be taken into account when defining strategies for surveillance, prevention and counselling.

Acknowledgements Luís Pacheco-Figueiredo received a grant from the Fundação para a Ciência e a Tecnologia (SFRH/SINTD/60124/2009).

Conflict of interest The authors declare that they have no conflicts of interest. 


\section{Appendix}

Table 2 Cumulative risk of metachronous second primary cancers and death, according to site of the first primary cancer and follow-up intervals

\begin{tabular}{|c|c|c|c|c|c|}
\hline \multirow[t]{2}{*}{ FPC } & \multirow{2}{*}{$\begin{array}{l}\text { Follow-up } \\
\text { (months) }\end{array}$} & \multicolumn{2}{|l|}{ Women } & \multicolumn{2}{|l|}{ Men } \\
\hline & & $\begin{array}{l}\text { Cumulative incidence } \\
\text { of metachronous SPC } \\
\%(95 \% \mathrm{CI})\end{array}$ & $\begin{array}{l}\text { Cumulative } \\
\text { risk of death } \\
\%(95 \% \mathrm{CI})\end{array}$ & $\begin{array}{l}\text { Cumulative incidence } \\
\text { of metachronous SPC } \\
\%(95 \% \mathrm{CI})\end{array}$ & $\begin{array}{l}\text { Cumulative risk } \\
\text { of death } \\
\%(95 \% \mathrm{CI})\end{array}$ \\
\hline \multirow[t]{3}{*}{ All } & 0 to $<12$ & $0.6(0.5-0.7)$ & $19.1(18.5-19.7)$ & $0.8(0.7-0.9)$ & $28.6(27.9-29.4)$ \\
\hline & $\geq 2$ to $<36$ & $1.6(1.4-1.8)$ & $32.9(32.1-33.8)$ & $2.2(2.0-2.4)$ & $45.4(44.5-46.3)$ \\
\hline & $\geq 2$ to $<60$ & $2.5(2.2-2.7)$ & $38.8(37.9-39.7)$ & $3.4(3.2-3.7)$ & $52.1(51.1-53.1)$ \\
\hline \multirow[t]{3}{*}{ Stomach (C16) } & 0 to $<12$ & $0.5(0.2-1.0)$ & $40.3(37.3-43.3)$ & $0.7(0.4-1.1)$ & $43.9(41.4-46.5)$ \\
\hline & $\geq 2$ to $<36$ & $1.3(0.8-1.9)$ & $57.6(54.2-61.3)$ & $1.9(1.4-2.5)$ & $61.7(58.8-64.8)$ \\
\hline & $\geq 2$ to $<60$ & $1.7(1.2-2.4)$ & $62.0(58.3-65.8)$ & $2.7(2.1-3.5)$ & $67.3(64.2-70.5)$ \\
\hline \multirow[t]{3}{*}{ Colon (C18) } & 0 to $<12$ & $0.7(0.4-1.2)$ & $20.5(18.5-22.7)$ & $0.7(0.4-1.2)$ & $20.6(18.7-22.6)$ \\
\hline & $\geq 2$ to $<36$ & $2.6(1.9-3.4)$ & $39.0(36.2-42.0)$ & $2.5(1.8-3.2)$ & $38.3(35.7-41.1)$ \\
\hline & $\geq 2$ to $<60$ & $4.0(3.1-5.0)$ & $46.2(43.2-49.5)$ & $3.8(3.0-4.7)$ & $47.4(44.5-50.5)$ \\
\hline \multirow[t]{3}{*}{ Rectum (C19 and C20) } & 0 to $<12$ & $1.0(0.5-1.8)$ & $19.2(16.5-22.2)$ & $1.5(0.9-2.3)$ & $16.9(14.7-19.3)$ \\
\hline & $\geq 2$ to $<36$ & $2.0(1.2-3.1)$ & $39.4(35.5-43.6)$ & $3.0(2.1-4.1)$ & $39.5(36.2-43.1)$ \\
\hline & $\geq 2$ to $<60$ & $2.9(1.9-4.2)$ & $47.7(43.5-52.4)$ & $4.2(3.1-5.4)$ & $48.3(44.7-52.3)$ \\
\hline \multirow[t]{3}{*}{ Lung (C34) } & 0 to $<12$ & $0.9(0.3-2.1)$ & $57.7(51.6-64.3)$ & $0.5(0.3-0.9)$ & $62.0(59.0-65.1)$ \\
\hline & $\geq 2$ to $<36$ & $1.8(0.9-3.3)$ & $77.7(70.5-85.3)$ & $0.9(0.6-1.4)$ & $84.2(80.7-87.8)$ \\
\hline & $\geq 2$ to $<60$ & $2.5(1.4-4.2)$ & $83.2(75.8-91.1)$ & $1.2(0.8-1.7)$ & $87.8(84.3-91.5)$ \\
\hline \multirow[t]{3}{*}{ Bladder (C67) } & 0 to $<12$ & $1.1(0.4-2.6)$ & $18.5(14.7-22.9)$ & $1.5(1.0-2.3)$ & $14.5(12.7-16.6)$ \\
\hline & $\geq 2$ to $<36$ & $2.7(1.4-4.7)$ & $31.3(26.3-37.0)$ & $3.8(2.9-4.9)$ & $28.6(26.0-31.4)$ \\
\hline & $\geq 2$ to $<60$ & $3.6(2.1-5.9)$ & $37.8(32.3-44.0)$ & $5.5(4.4-6.8)$ & $35.2(32.3-38.3)$ \\
\hline \multirow[t]{3}{*}{ Breast (C50) } & 0 to $<12$ & $0.2(0.1-0.3)$ & $3.7(3.2-4.3)$ & & \\
\hline & $\geq 2$ to $<36$ & $1.2(0.9-2.5)$ & $12.9(11.9-14.0)$ & & \\
\hline & $\geq 2$ to $<60$ & $2.2(1.8-2.7)$ & $19.4(18.1-20.6)$ & & \\
\hline \multirow[t]{3}{*}{ Prostate (C61) } & 0 to $<12$ & & & $0.7(0.5-1.0)$ & $5.5(4.8-6.2)$ \\
\hline & $\geq 2$ to $<36$ & & & $2.6(2.2-3.1)$ & $16.5(15.3-17.7)$ \\
\hline & $\geq 2$ to $<60$ & & & $4.5(3.9-5.1)$ & $25.0(23.5-26.4)$ \\
\hline
\end{tabular}

$F P C$ first primary cancers, SPC second primary cancers, $95 \%$ CI $95 \%$ confidence interval

${ }^{a}$ Metachronous second primary cancers are those diagnosed in the period $\geq 2$ to $<12$ months after the diagnosis of the first primary cancer

\section{References}

1. Araujo F, et al. Trends in cardiovascular diseases and cancer mortality in 45 countries from five continents (1980-2010). Eur J Prev Cardiol. 2013.

2. De Angelis R et al. Cancer survival in Europe 1999-2007 by country and age: results of EUROCARE-5 - a population-based study. Lancet Oncol. 2014;15(1):23-34.

3. Ferlay J et al. GLOBOCAN 2012 v1.0, cancer incidence and mortality worldwide. Lyon: International Agency for Research on Cancer; 2013.

4. Pacheco-Figueiredo L, Lunet N. Health status, use of healthcare, and socio-economic implications of cancer survivorship in Portugal: results from the Fourth National Health Survey. J Cancer Surviv. 2014.
5. Curtis R, et al. New malignancies among cancer survivors: SEER cancer registries, 1973-2000. National Cancer Institute; NIH Publ., 2006. No. 05-5302.

6. Travis LB. The epidemiology of second primary cancers. Cancer Epidemiol Biomarkers Prev. 2006;15(11):2020-6.

7. Rosso $\mathrm{S}$ et al. Multiple tumours in survival estimates. Eur J Cancer. 2009;45(6):1080-94.

8. Pacheco-Figueiredo L et al. Evaluation of the frequency of and survival from second primary cancers in North Portugal: a population-based study. Eur J Cancer Prev. 2013;22(6):599-606.

9. International rules for multiple primary cancers (ICD-0 third edition). Eur J Cancer Prev, 2005; 14(4):307-8.

10. Howe HL. A review of the definition for multiple primary cancers in the United States. Springfield: North American Association of Central Cancer Registries; 2003. 
11. RORENO. Registo Oncologico Regional do Norte. IPO - Porto. 2006.

12. Teppo L, Pukkala E, Saxen E. Multiple cancer - an epidemiologic exercise in Finland. J Natl Cancer Inst. 1985;75(2):207-17.

13. Youlden DR, Baade PD. The relative risk of second primary cancers in Queensland, Australia: a retrospective cohort study. BMC Cancer. 2011;11:83.

14. Jegu J et al. The effect of patient characteristics on second primary cancer risk in France. BMC Cancer. 2014;14:94.

15. Tabuchi $\mathrm{T}$ et al. Incidence of metachronous second primary cancers in Osaka, Japan: update of analyses using population-based cancer registry data. Cancer Sci. 2012;103(6):1111-20.

16. Storm HH et al. Multiple primary cancers in Denmark 1943-80; influence of possible underreporting and suggested risk factors. Yale J Biol Med. 1986;59(5):547-59.

17. Coleman MP. Multiple primary malignant neoplasms in England and Wales, 1971-1981. Yale J Biol Med. 1986;59(5):517-31.

18. Crocetti E, Buiatti E, Falini P. Multiple primary cancer incidence in Italy. Eur J Cancer. 2001;37(18):2449-56.

19. Liu L et al. Prevalence of multiple malignancies in the Netherlands in 2007. Int J Cancer. 2011;128(7):1659-67.

20. Nielsen SF, Nordestgaard BG, Bojesen SE. Associations between first and second primary cancers: a population-based study. CMAJ. 2012;184(1):E57-69.

21. Morrison A. Screening in chronic disease. New York: Oxford University Press; 1985.

22. Cancer trends progress report-2011/2012 update. National Cancer Institute, NIH Publ., 2012.

23. Corkum $\mathrm{M}$ et al. Screening for new primary cancers in cancer survivors compared to non-cancer controls: a systematic review and meta-analysis. J Cancer Surviv. 2013;7(3):455-63.
24. Pacheco-Figueiredo $\mathrm{L}$ et al. Health-related behaviours in the EpiPorto study: cancer survivors versus participants with no cancer history. Eur J Cancer Prev. 2011;20(4):348-54.

25. Rundle A et al. A prospective study of socioeconomic status, prostate cancer screening and incidence among men at high risk for prostate cancer. Cancer Causes Control. 2013;24(2):297-303.

26. Morgan RM et al. Socioeconomic variation and prostate specific antigen testing in the community: a United Kingdom based population study. J Urol. 2013;190(4):1207-12.

27. Henderson BE, Feigelson HS. Hormonal carcinogenesis. Carcinogenesis. 2000;21(3):427-33.

28. Thompson D, Easton DF. Cancer incidence in BRCA1 mutation carriers. J Natl Cancer Inst. 2002;94(18):1358-65.

29. Mavaddat $\mathrm{N}$ et al. Cancer risks for BRCA1 and BRCA2 mutation carriers: results from prospective analysis of EMBRACE. J Natl Cancer Inst. 2013;105(11):812-22.

30. Dourado F, Carreira H, Lunet N. Mammography use for breast cancer screening in Portugal: results from the 2005/2006 National Health Survey. Eur J Pub Health. 2013;23(3):386-92.

31. Shafer D, Albain K. Lung cancer outcomes in women. Semin Oncol. 2009;36(6):532-41.

32. Alves L, Bastos J, Lunet N. Trends in lung cancer mortality in Portugal (1955-2005). Rev Port Pneumol. 2009;15(4):575-87.

33. Carreira $\mathrm{H}$ et al. Trends in the prevalence of smoking in Portugal: a systematic review. BMC Public Health. 2012;12:958.

34. Castro $\mathrm{C}$ et al. Assessing the completeness of cancer registration using suboptimal death certificate information. Eur J Cancer Prev. 2012;21(5):478-9.

35. Travis LB et al. Aetiology, genetics and prevention of secondary neoplasms in adult cancer survivors. Nat Rev Clin Oncol. 2013;10(5):289-301. 